\title{
Effects of Zinc on Non-alcoholic Fatty Liver Disease After Pancreatoduodenectomy
}

\author{
Atsushi Miyoshi ${ }^{1, ~}{ }^{*}$, Hiroki Koga ${ }^{1}$, Satomi Nakamura ${ }^{1}$, Hiroaki Nakamura ${ }^{1}$, Kohei Yamada ${ }^{1}$, \\ Hiroshi Kubo ${ }^{1}$, Masatsugu Hiraki ${ }^{1}$, Osamu Ikeda ${ }^{1}$, Toshiya Tanaka ${ }^{1}$, Kenji Kitahara ${ }^{1}$, Seiji Sato ${ }^{1}$, \\ Hirokazu Noshiro ${ }^{2}$
}

${ }^{1}$ Department of Surgery, Saga-Ken Medical Centre Koseikan, Saga, Japan

${ }^{2}$ Department of Surgery, Faculty of Medicine, Saga University, Saga, Japan

Email address:

miyoshi-a@koseikan.jp (A. Miyoshi)

${ }^{*}$ Corresponding author

\section{To cite this article:}

Atsushi Miyoshi, Hiroki Koga, Satomi Nakamura, Hiroaki Nakamura, Kohei Yamada, Hiroshi Kubo, Masatsugu Hiraki, Osamu Ikeda, Toshiya Tanaka, Kenji Kitahara, Seiji Sato, Hirokazu Noshiro. Effects of Zinc on Non-alcoholic Fatty Liver Disease After Pancreatoduodenectomy. Journal of Surgery. Vol. 7, No. 5, 2019, pp. 148-153. doi: 10.11648/j.js.20190705.17

Received: August 17,2019; Accepted: September 12, 2019; Published: September 26, 2019

\begin{abstract}
Background: The etiology, treatment and prevention of Nonalcoholic fatty liver disease (NAFLD) after pancreatoduodenectomy (PD) remain largely unknown. We aimed to elucidate the risk factors for NAFLD after PD and investigate the nutritional effects of zinc medication. Methods: We retrospectively examined 109 patients who underwent PD between 2013 and 2017. We diagnosed the postoperative NAFLD using CT attenuation at six months later. We identified the risk factors for postoperative NAFLD among perioperative factors and analyzed the nutritional effect of zinc medication at six months after surgery. Results: We diagnosed 27 patients with NAFLD after PD. A univariate analysis showed that pancreas cancer $(p=0.029)$, operative time $(p=0.008)$, blood loss $(p=0.034)$, postoperative diarrhea $(p<0.001)$ and zinc medication $(\mathrm{p}<0.001)$ were associated with postoperative NAFLD. A multivariate analysis demonstrated that zinc medication was the most important factor for the prevention of NAFLD after PD. All patients who received zinc also took pancreatic enzyme simultaneously and showed a significantly lower rate of body weight loss than in patients without zinc at six months after PD $(p=0.041)$. These patients showed a significantly higher total cholesterol level $(p=0.006)$ and higher serum zinc level $(\mathrm{p}<0.001)$. Furthermore, significantly fewer cases of postoperative NAFLD were noted among the patients who received zinc than among those who did not receive it $(5.7 \%$ vs $33.8 \%: \mathrm{p}=0.001)$. Conclusion: These results suggest that zinc administration might improve the postoperative nutritional status and prevent NAFLD after PD.
\end{abstract}

Keywords: Pancreatoduodenectomy, Nonalcoholic Fatty Liver Disease, Zinc

\section{Introduction}

Pancreatoduodenectomy (PD) has become the standard operation for patients with peri-ampullary disease. The longterm survival after PD has gradually increased due to recent improvements in surgical techniques and perioperative management [1]. However, the extensive resection of organs and complicated reconstruction of the alimentary tract associated with PD lead to the inhibition of the pancreatic function and malnutrition in most patients [2]. Therefore, postoperative nutritional management is extremely important for patients undergoing PD.
Recently, reports of nonalcoholic fatty liver disease (NAFLD) after PD with malnutrition has become increasingly problematic and few reports documented some cases of hepatic failure after pancreatic resection [3-7]. However, the clinicopathological and etiological findings of NAFLD after PD are largely unknown. A few reports have suggested that NAFLD after PD was associated with postoperative malnutrition caused by pancreatic exocrine sufficiency $[8,9]$. Other reports have suggested that the supplementation of pancreatic enzyme, such as pancrealipase, was effective for the treatment and prevention of NAFLD after PD $[10,11]$.

In addition, zinc deficiency has been occasionally reported 
in patients who have undergone PD and is said to be closely correlated with the pancreatic exocrine function [12, 13]. Polaprezinc consists of zinc and has been reported to attenuate liver fibrosis in non-alcoholic steatohepatitis [14, 15].

In this study, we examined the risk factors for NAFLD after PD and investigated the nutritional effect of the supplementation of zinc.

\section{Methods}

\subsection{Patients}

This retrospective study included 109 consecutive patients who underwent pancreatoduodenectomy between April 2013 and December 2017 at the Department of Surgery, Saga-Ken Medical Centre Koseikan, Japan. Table 1 shows the patient characteristics of this study. The mean age was 68.5 years old, and 64 males and 45 females were included. The mean body weight and body mass index (BMI) were $56.4 \mathrm{~kg}$ and 22.2 $\mathrm{kg} / \mathrm{m}^{2}$, respectively. The histological diagnosis was pancreatic carcinoma in $45(41.3 \%)$, distal bile duct carcinoma in 27 (24.8\%), ampulla of Vater carcinoma in $16(14.7 \%)$, Intraductal papillary mucinous neoplasm (IPMN) in $12(11.0 \%)$, pancreatic neuroendocrine tumor in $4(3.7 \%)$, and others in $5(4.5 \%)$. Regarding the surgical procedures, subtotal stomach-preserving PD and conventional PD were performed in 102 (93.5\%), and 7 $(6.5 \%)$ patients, respectively. Portal vein resection was performed in $30(29.4 \%)$ patients who were suspected of having direct invasion to the vein.

Table 1. Patient characteristics $(n=109)$

\begin{tabular}{ll}
\hline Age (years) & $68.5 \pm 8.6$ \\
Gender (male/female) & $64 / 45$ \\
Body weight (kg) & $56.4 \pm 9.8$ \\
BMI $\left(\mathrm{kg} / \mathrm{m}^{2}\right)$ & $22.2 \pm 3.0$ \\
Diagnosis & \\
Pancreas cancer & $45(41.3 \%)$ \\
Distal bile duct cancer & $27(24.8 \%)$ \\
Ampulla of Vater cancer & $16(14.7 \%)$ \\
IPMN & $12(11.0 \%)$ \\
PNET & $4(3.7 \%)$ \\
Others & $5(4.5 \%)$ \\
Operation & \\
SSPPD & $102(93.5 \%)$ \\
PD & $7(6.5 \%)$ \\
Operation time (min.) & $394 \pm 92$ \\
Blood loss (ml) & $803 \pm 658$ \\
Portal vein resection & $30(29.4 \%)$ \\
Postoperative morbidity & \\
DGE & $4(3.6 \%)$ \\
Pancreatic fistula & $16(14.8 \%)$ \\
(ISGPF $\geq$ Grade B) & \\
Mortality & $0(0)$ \\
\hline
\end{tabular}

BMI: body mass index, IPMN: intraductal papillary mucinous neoplasm, PNET: pancreatic neuroendocrine tumor, SSPPD: subtotal stomach preserving pancreatoduodenectomy, PD: pancreatoduodenectomy, DGE: delayed gastric empty, ISGPF: international study group of pancreatic fistula

To prevent postoperative severe diarrhea, dissection of the nerve plexus around the superior mesenteric artery (SMA) was avoided and the cutting line of pancreas was on the portal vein in most patients. The reconstruction procedure was a modified Child's method that involved end-to-side pancreaticojejunostomy plus Braun anastomosis with external drainage of the main pancreatic duct in most patients. Postoperative supplementation therapy including pancreatic enzyme (pancrealipase; Eisai Pharmaceutical Co., Japan), or zinc (polaprezinc; Zeria Pharmaceutical Co., Tokyo, Japan) was prescribed to patients at the surgeon's discretion. The dosages of Pancrealipase were $900 \mathrm{mg}$ $1800 \mathrm{mg} /$ day and those of polaprezinc were $75 \mathrm{mg}$ /day that contain $34 \mathrm{mg}$ of zinc. Postoperative adjuvant chemotherapy with S-1 (Taiho Pharmaceutical Co, Tokyo, Japan) was usually administered to patients with pancreas carcinoma and bile duct carcinoma for six months.

All patients underwent periodic abdominal computed tomography (CT) and were evaluated the postoperative nutritional status at the blood test. The routine follow-up interval was once every three to four months.

Informed consent was obtained from all individual participants included in the study.

This study was approved as project number 17-11-3-1 by the Ethics Committee of Saga-Ken Medical Centre Koseikan, and conducted in accordance with mandates of the Helsinki Declaration.

\subsection{The Diagnosis of Postoperative NAFLD Using CT Attenuation}

CT images were obtained with a 64-multidetector CT scanner, and the raw data set was reconstructed at 2-mm thickness. At six months after surgery, the average CT attenuation values in four sectors of the liver and in one region of the spleen were monitored to determine the hepatic fat content for each patient. We calculated the liver-to-spleen attenuation ratio (L/S ration) on $\mathrm{CT}$, and postoperative NAFLD was defined as an L/S ratio of $<0.9[16,17]$. Four patients with NAFLD after PD were underwent the liver biopsy and diagnosed by the pathological findings.

\subsection{Analyses of the Risk Factors for NAFLD}

We evaluated the pre-, intra-, and postoperative factors that might be associated with the development of NAFLD after PD. The preoperative factors were age, gender, BMI, preoperative jaundice, diabetes mellitus, and primary disease. The intraoperative factors were operative time, the volume of blood loss, pancreatic consistency, the resection of portal vein, and total pancreatectomy. The postoperative factors were postoperative enteral nutrition, the presence of pancreatic fistula based on the International Study Group on Pancreatic Fistula criteria [18], eating disorder, and diarrhea based on Common Terminology Criteria for Adverse Events (CTCAE), version 4.0. Other postoperative factors included the administration of adjuvant chemotherapy, pancrealipase, polaprezinc, and insulin.

\subsection{Analyses of the Effect of Zinc in PD Patients}

We analyzed the nutritional effect of zinc administration on 
patients after PD. We evaluated the postoperative nutritional state, such as BMI loss, body weight (BM) loss, BM loss rate calculated by (preoperative BM- postoperative BM) / preoperative $\mathrm{BM} \times 100$, serum albumin, transaminase, total cholesterol, and serum zinc levels at six months after surgery.

\subsection{Statistical Analyses}

Continuous variables were expressed as the mean ( \pm standard deviation) and compared using Student's $t$-test. Categorical variables were compared using the $\chi^{2}$-test. A P value of less than 0.05 was considered statistically significant.

Variables associated with the development of postoperative NAFLD were first assessed using a univariate analysis. The variables that were significant were then subjected to a multivariate logistic regression analysis to identify the independent risk factors for the development of postoperative NAFLD after PD.

All data were analyzed using the StatView software program, version 5.0 (SAS Institute, Cary, NC, USA) and the SPSS 15.0 software program (SPSS Chicago. IL, USA).

\section{Results}

\subsection{Risk Factors for NAFLD After PD}

We diagnosed $27(24.8 \%)$ patients with NAFLD in this series. Table 2 shows the results of the univariate analysis for identifying the risk factors associated with the NAFLD after PD. Among pre- and intraoperative factors, a primary disease of pancreas cancer $(p=0.029)$, the operative time $>400$ minutes $(\mathrm{p}=0.008)$, and blood loss $>500 \mathrm{ml}(\mathrm{p}=0.034)$ were found to be significantly correlated with NAFLD after PD. Postoperative NAFLD was not associated with the administration of pancrealipase. However, postoperative diarrhea $(\mathrm{p}<0.001)$ and the administration of polaprezinc $(\mathrm{p}$ $<0.001)$ were correlated with NAFLD after PD.

Table 2. Univariate analysis of the risk factors for NAFLD after PD.

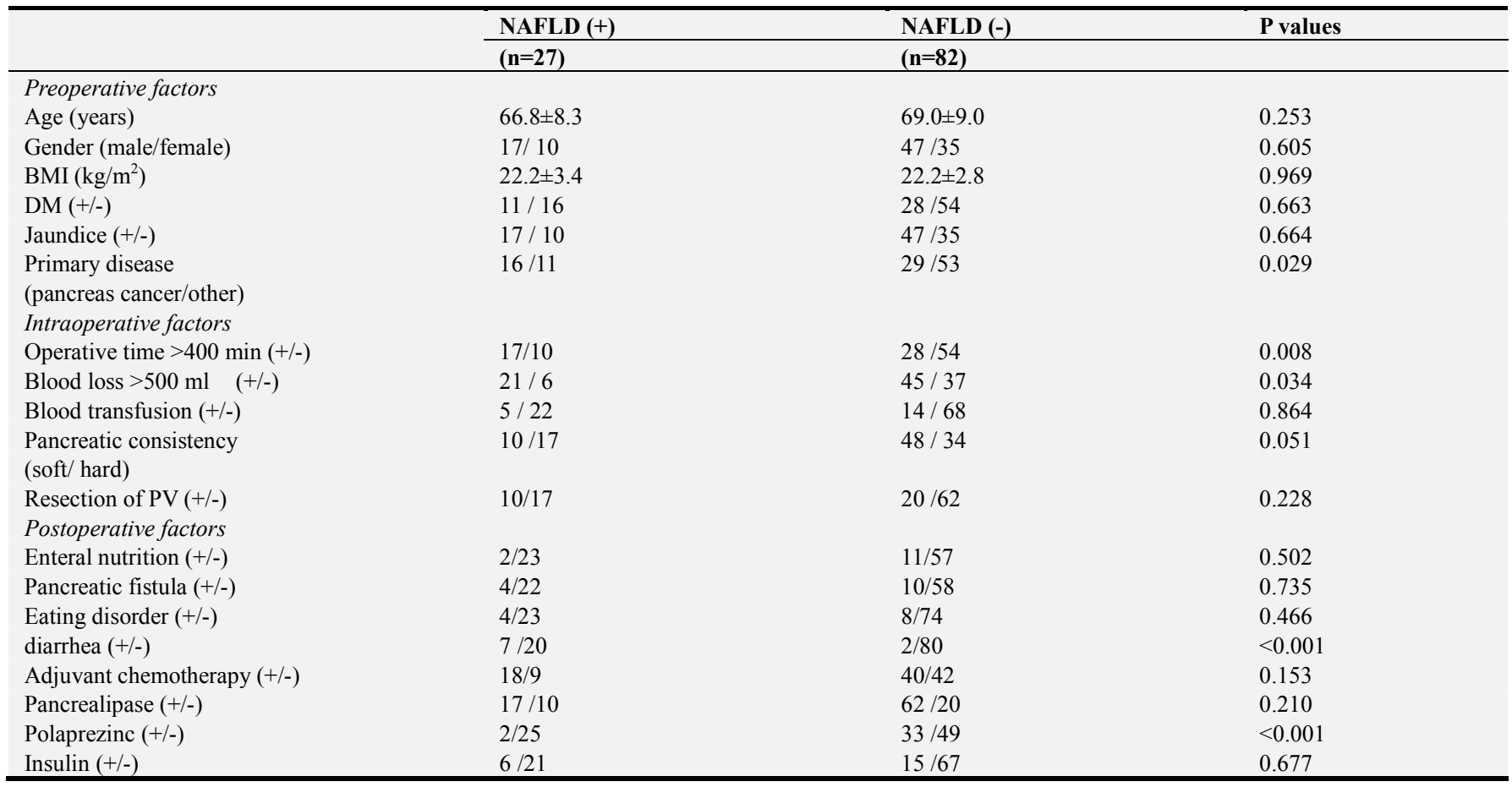

NAFLD: nonalcoholic fatty liver disease, BMI: body mass index, DM: diabetes mellitus, PV: portal vein

Table 3 shows the results of the multivariate analysis for identifying the independent risk factors for NAFLD after PD. Postoperative diarrhea and polaprezinc were independent predictors of postoperative NAFLD.

Table 3. Multivariate analysis of the risk factors for NAFLD after PD.

\begin{tabular}{llll}
\hline Factor & Hazard ratio & $\mathbf{9 5 \%}$ CI & P value \\
\hline Pancreas cancer & 2.260 & $0.784-6.510$ & 0.131 \\
Operating time $>400$ min & 2.028 & $0.642-6.399$ & 0.228 \\
Blood loss $>500 \mathrm{ml}$ & 1.733 & $0.480-6.258$ & 0.8401 \\
Diarrhea & 14.962 & $2.099-106.634$ & 0.006 \\
Polaprezinc & 10.416 & $0.016-0.561$ & 0.009 \\
\hline
\end{tabular}

CI: confidence interval

\subsection{Effects of Zinc Administration on PD Patients}

Table 4 shows the analysis of the nutritional effect of zinc on patients at 6 months after PD. In the background of patients, all patients who received polaprezinc also took pancrealipase simultaneously $(\mathrm{p}<0.001)$. However, there was no significant differences in the other background factors. In the nutritional evaluation, BMI and BM loss in patients with polaprezinc were less than in patients without polaprezinc. BM loss rate in patients with polaprezinc was significantly lower than in patients without polaprezinc (0.041). Patients who received polaprezinc had significantly lower ALT levels $(\mathrm{p}=0.014)$, higher total cholesterol levels $(p=0.004)$, and higher serum zinc levels $(p<$ 
0.001). Furthermore, significantly fewer cases of postoperative NAFLD were noted among the patients who received polaprezinc than among those who did not receive it $(5.7 \%$ vs $33.8 \%: \mathrm{p}=0.001)$.

Table 4. An analysis of the nutritional effect of zinc.

\begin{tabular}{|c|c|c|c|}
\hline \multirow{2}{*}{ Factor } & \multirow{2}{*}{$\begin{array}{l}\text { Polaprezinc }(+) \\
(n=35)\end{array}$} & \multirow{2}{*}{$\begin{array}{l}\text { Polaprezinc (-) } \\
(n=74)\end{array}$} & \multirow{2}{*}{ P value } \\
\hline & & & \\
\hline \multicolumn{4}{|l|}{ Background } \\
\hline Gender (male/female) & $19 / 16$ & $45 / 29$ & 0.519 \\
\hline Age & $68.8 \pm 7.9$ & $68.3 \pm 9.0$ & 0.772 \\
\hline Primary disease (pancreas ca/other) & $15 / 20$ & $30 / 44$ & 0.818 \\
\hline Portal vein resection $(+/-)$ & $9 / 26$ & $21 / 53$ & 0.901 \\
\hline Adjuvant chemotherapy (+/-) & $19 / 16$ & $39 / 35$ & 0.575 \\
\hline Pancrealipase (+/-) & $35 / 0$ & $44 / 30$ & $<0.001$ \\
\hline Insulin $(+/-)$ & $5 / 30$ & $16 / 57$ & 0.338 \\
\hline \multicolumn{4}{|l|}{ Nutrition } \\
\hline BMI loss $\left(\mathrm{kg} / \mathrm{m}^{2}\right)$ & $-1.6 \pm 1.6$ & $-2.2 \pm 1.9$ & 0.114 \\
\hline Body weight loss (kg) & $-4.2 \pm 3.5$ & $-5.7 \pm 4.3$ & 0.094 \\
\hline Rate of Body weight loss (\%) & $6.4 \pm 7.0$ & $9.4 \pm 7.3$ & 0.041 \\
\hline Diarrhea $(+/-)$ & $2 / 33$ & $7 / 67$ & 0.507 \\
\hline Eating disorder $(+/-)$ & $5 / 30$ & $7 / 67$ & 0.452 \\
\hline $\operatorname{AST}(\mathrm{U} / \mathrm{L})$ & $26.1 \pm 10.8$ & $38.4 \pm 46.3$ & 0.123 \\
\hline $\operatorname{ALT}(\mathrm{U} / \mathrm{L})$ & $19.8 \pm 8.8$ & $36.5 \pm 39.2$ & 0.014 \\
\hline Albumin (g/dL) & $3.8 \pm 0.4$ & $3.6 \pm 0.6$ & 0.166 \\
\hline Cholinesterase (U/L) & $241.8 \pm 86.1$ & $218.2 \pm 89.5$ & 0.229 \\
\hline Total cholesterol (mg/dL) & $163.2 \pm 44.7$ & $138.6 \pm 32.8$ & 0.004 \\
\hline Zinc $(\mu \mathrm{g} / \mathrm{dL})$ & $102.8 \pm 41.2$ & $66.4 \pm 16.0$ & $<0.001$ \\
\hline Postoperative NAFLD & $2(5.7 \%)$ & $25(33.8 \%)$ & 0.001 \\
\hline
\end{tabular}

BMI: body mass index, AST: aspartate aminotransferase, ALT: alanine aminotransferase, NAFLD: nonalcoholic fatty liver disease

\section{Discussion}

Recently, NAFLD after PD with malnutrition has become increasingly problematic. Studies exploring the mechanism of postoperative NAFLD have reported several risk factors, including pancreas cancer, postoperative diarrhea, blood loss, small remnant pancreatic volume, and a long operative time $[5,6,19]$.

Several reports have suggested that NAFLD after PD is mainly associated with pancreatic exocrine dysfunction [5. 6. 9]. Nakagawa et al showed that postoperative pancreatic exocrine sufficiency was an independent risk factor for NAFLD [8]. Nagai et al and Kishi et al demonstrated that the administration of pancrealipase was useful for the treatment of NAFLD after PD [10, 11]. However, Satoi et al failed to show a significant preventive effect of pancrealipase for NAFLD development after PD on randomized control trial [20].

In the current study, we analyzed the risk factors for NAFLD after PD. The univariate analysis revealed that postoperative NAFLD was associated with pancreas cancer, the operative time, blood loss, postoperative diarrhea, and the administration of polaprezinc but not pancrealipase. The multivariate analysis identified postoperative diarrhea and polaprezinc as independent predictive factors for NAFLD after PD. These analyses demonstrated that administration of zinc was the most important crucial factor for the prevention of NAFLD after PD.

Zinc is the most abundant intracellular trace element and is strongly implicated in cell turnover and repair systems. Indeed, zinc deficiency has been reported to increase the inflammatory conditions and disrupt the barrier function of endothelial cells $[21,22]$. PD with massive resection of the pancreas causes a zinc deficiency, as zinc is absorbed mainly in the distal duodenum and proximal jejunum. In addition, zinc deficiency after PD has been reported to impair the pancreatic exocrine functions and cause maldigestion, malabsorption, and malnutrition [23, 24]. Kato et al reported that a high-zinc diet after major pancreatectomy enhanced cell proliferation in the remnant pancreas and improved the pancreatic exocrine function in a dog model [12]. However, the usefulness of zinc for NAFLD after PD has not been elucidated in the clinical data.

Polaprezinc consists of zinc and carnosine and is used for the treatment of gastric ulcers. Zinc and carnosine are reactive oxygen species (ROS) scavengers and have antioxidant activities [25, 26]. Polaprezinc was reported to attenuate liver fibrosis in conventional non-alcoholic steatohepatitis by reducing inflammation and lipid peroxidation in a mouse model [14]. However, no study has investigated the effect of polaprezinc on NAFLD after PD in a clinical setting.

In the present study, most patients with zinc had a wellcontrolled nutritional status after PD and showed a significantly lower BM loss rate and higher serum zinc level and total cholesterol level than those without zinc. Furthermore, almost no patients with zinc presented with postoperative NAFLD. These results suggested that zinc administration might improve the postoperative nutritional status and prevent NAFLD after PD. In addition, all patients with zinc simultaneously took pancrealipase. Therefore, both zinc and pancreatic enzymes might be important for preventing postoperative NAFLD. Oxidative stress has also 
been reported to play a central role in the pathogenesis of NAFLD [27, 28]. The effect of polaprezinc as an ROS scavenger might help prevent NAFLD development.

This retrospective analysis was informative butt had some limitations. First, postoperative supplementation therapy including pancreatic enzyme or zinc was prescribed to patients at each surgeon's discretion. Second, the postoperative pancreatic exocrine function, which was associated with zinc, was not examined. Therefore, a prospective randomized control study including an analysis of the pancreatic exocrine function should be considered to elucidate the prevention effect of zinc for NAFLD after PD.

\section{Conclusion}

The postoperative diarrhea and the zinc administration are independent predictors of postoperative NAFLD. Zinc administration might improve the postoperative nutritional status and prevent NAFLD after PD.

This is the first study to demonstrate the prevention effect of zinc for NAFLD after PD using clinical data. To prevent NAFLD after PD, the administration of zinc, pancreatic enzyme usage, and provision of adequate nutritional support are recommended.

\section{References}

[1] Murakami Y, Uemura K, Hayashidani Y, Sudo T, Hashimoto $\mathrm{Y}$, Nakagawara N, et al. No mortality after 150 consecutive pancreatoduodenectomies with duct-to-mucosa pancreaticogastrostomy. J Surg Oncol 2008; 97: 205-209.

[2] Fish JC, Smith LB, Williams RD Digestive function after radical pancreaticoduodenectomy. Am J Surg 1969; 117: 4045 .

[3] Nomura R, Ishizaki Y, Suzuki K, Kawasaki S. Development of hepatic steatosis after pancreatoduodenectomy. Am J Roentgenol 2007; 189: 1484-1488.

[4] Yu HH, Shan YS, Lin PW Effect of pancreatticoduodenectomy on the course of hepatic steatosis. World J Surg 2010; 34: 2122-2127.

[5] Kato H, Isaji S, Azumi Y, Kishiwada M, Hamada T Mizuno S, et al. Development of nonalcoholic fatty liver disease (NAFLD) and nonalcoholic steatohepatitis (NASH) after pancreaticoduodenectomy: Proposal of a postoperative NAFLD scoring system. J Hepatobiliary Pancreat Sci 2010; 17: $296-304$.

[6] Tanaka N, Horiuchi A, Yokoyama T, Kaneko G, Horigome N, Yamura $\mathrm{T}$, et al. Clinical characteristics of de novo nonalcoholic fatty liver disease following pancreaticoduodenectomy. J Gastroenterol 2011; 46: 758-768.

[7] Sim EH, Kwon JH, Kim SY, Jung SM, Maeng LS, Jang JW, et al. Severe steatohepatitis with hepatic decompensation resulting from malnutrition after pancreaticoduodenectomy. Clin Mol Hepatol 2012; 18: 404-410.

[8] Nakagawa N, Murakami Y, Uemura K, Sudo T, Hashimoto Y, Kondo N, et al. Nonalcoholic fatty liver disease after pancreatoduodenectomy is closely associated with postoperative pancreatic exocrine insufficiency. J Surg Oncol 2014; 110: 720-726.

[9] Murata Y, Mizuno S, Kato H, Kishiwada M, Ohsawa I, Hamada $\mathrm{T}$, et al. Nonalcoholic steatohepatitis (NASH) after pancreaticoduodenectomy: association of pancreatic exocrine deficiency and infection. Clin J Gastroenterol 2011; 4: 242-248.

[10] Nagai M, Sho M, Satoi S, Toyokawa H, Akahori T, Yanagimoto $\mathrm{H}$, et al. Effect of pancrelipase on nonalcoholic fatty liver disease after pancreaticoduodenectomy. J Hepatobililary Pancreat Sci 2014; 21: 186-192.

[11] Kishi Y, Shimada K, Nara S, Esaki M, Kosuge T. Administration of pancrealipase as effective treatment for hepatic steatosis after pancreatectomy. Pancreas 2015; 44: 983-987.

[12] Kato K, Isaji S, Kawarada Y, Hibasami H, Nakashima K. Effect of zinc administration on pancreatic regeneration after $80 \%$ pancreatectomy. Pancreas 1996; 14: 158-165.

[13] Yu HH, Yang TM, Shan YS, Lin PW. Zinc deficiency in patients undergoing pancreatoduodenectomy for periampullary tumors is associated with pancreatic exocrine insufficiency. World J Surg 2011; 35: 2110-2117.

[14] Sugino H, Kumagai N, Watanabe S, Toda K, Takeuchi O, Tsunematsu S, et al. Polaprezinc attenuates liver fibrosis in a mouse model of non-alcoholic steatohepatitis. J Gastroenterol Hepatol 2008; 23: 1909-1916.

[15] Matsuoka S, Matsumura H, Nakamura H, Oshiro S, Arakawa $\mathrm{Y}$, Hayashi $\mathrm{J}$, et al. Zinc supplementation improves the outcome of chronic hepatitis $\mathrm{C}$ and liver cirrhosis. J Clin Biochem Nutr 2009; 45: 292-303.

[16] Kawamoto S, Soyer PA, Fishman EK, Bluemke DA. Nonneoplatstic liver disease: Evaluation with CT and MRI imaging. Radiographics 1998; 18: 827-848.

[17] Park SH, Kim PN, Kim KW, LEE SW, Yoon SE, Park SW, et al. Macrovesicular hepatic steatosis in living liver donors: Use of CT for quantitative and qualitative assessment. Radiology 2006; 239: 105-112.

[18] Shrikhande SV, Sivasanker M, Vollmer CM, Friess H, Besselink MG, Fingerhut A, et al. International Study Group of Pancreatic Surgery (ISGPS) . Pancreatic anastomosis after pancreatoduodenectomy: A position statement by the International Study Group of Pancreatic Surgery (ISGPS) 2017; 161: 1221-1234.

[19] Sato T, Matsuo Y, Shiga K, Morimoto M Miyai H, Takeyama $\mathrm{H}$. Factors that predict the occurrence of and recovery from non-alcoholic fatty liver disease after pancreatoduodenectomy. Surgery 2016; 160: 318-330.

[20] Satoi S, Sho M, Yanagimoto H, Yamamoto T, Akahori T, Kinoshita S, et al. Do pancrelipase delayed-release capsules have a protective role against nonalcoholic fattyliver disease after pancreatoduodenectomy in patients with pancreatic cancer? A randomized controlled trial. J Hepatobililary Pancreat Sci 2016; 23: 167-173.

[21] Sturniolo Gc, Fries W, Mazzon E, Di Leo V, Barollo M, D'inca R. Effect of zinc supplementation on intestinal permeability in experimental colitis. J Lab Clin Med 2002; 139: 311-315. 
[22] Henning B, Wang Y, Ramasamy S, McClain CJ. Zinc deficiency alters barrier function of cultured porcine endothelial cells. J Nutr 1992; 122: 1242-1247.

[23] Kim YJ, Kim MY, Kim HO, Lee MD, Park YM. Acrodematitis enteropathica-like eruption associated with combined nutritional deficiency. J Korean Med Sci2005; 20: 908-911.

[24] Dutta SK, Procaccino F, Aamodt R Zinc metabolism in patienyts with exocrine pancreatic insufficiency. J Am Coll Nutr 1998; 17: 556-563.

[25] Powell SR. The antioxidant properties of zinc. J Nutr 2000; 130: $1447 \mathrm{~S}-1254 \mathrm{~S}$.
[26] Yoshikawa T, Naito Y, Tanigawa T, Yoneta T, Kondo M. The antioxidant properties of a novel zinc-carnosine chelate compound, N- (3-aminopropionyl) -L-histidinato zinc. Biochem Biophys Acta 1991; 1115: 15-22.

[27] Tilg H, Diehl AM. Cytokines in alcoholic and nonalcoholic steatohepatitis. N Engl J Med. 2000; 343: 1467-1476.

[28] Kitade H, Chen G, Ni Y, Ota T. Nonalcoholic fatty liver disease and insulin resistance: New insights and potential new treatment. Nutrients 2017; 14: 9. 